\title{
Greenhouse gas emissions and energy use of self-selected diet is not associated with diet quality among Japanese adults
}

\section{Abstract}

Many previous studies supported that shift of dietary choice is necessary to reduce environmental impact and achieve a healthy diet. However, inconsistent results have been shown for the association between the environmental impact of diet and its nutritional adequacy. The aim of this study was to investigate the association between environmental indicator including greenhouse gas emissions (GHGE) and energy use (EU) and diet quality using several diet quality scores among Japanese. Dietary data were obtained from a cross-sectional study including 392 healthy adults (196 women and 196 men, aged 20-69 y) living in 20 areas of 47 prefectures, in which four non-consecutive-day diet record was collected from February to March in 2013. Diet-related GHGE and EU were estimated using the Global Link Input-Output model. Diet quality was assessed with the adherence to the Japanese Food Guide Spinning Top (JFG score), its modified version (modified JFG score), Nutrient-rich Dietary index (NRD9.3.), the Mediterranean diet score (MDS) and the Dietary Approaches to Stop Hypertension (DASH) score. Participants were categorized into sex-specific tertiles according to energy-adjusted GHGE or EU then mixed for the analysis. Using the PROC GLM procedure, linear regression models were constructed to examine the association of environmental indicators with diet quality and intakes of foods and nutrition. Diet-related GHGE and EU were positively associated with NRF.9.3 score and MDS. Diet-related EU was also positively associated with DASH score. However, there was no significant association observed in either JFG score or the modified JFG score with both diet-related GHGE and EU. Diet-related GHGE and EU were both negatively associated with intakes of well-milled rice, fats and oils, and sugar-sweetened beverage, and positively associated with intakes of vegetables, mushroom, fish and seafood, tea and coffee and seasoning, protein, and several micronutrients. A positive association between GHGE or EU, and NRF.9.3 or MSD was seemed to be due to higher intake of vegetables, fish and seafood, meat, vitamin A, iron, potassium in high GHGE or EU group than low GHGE or EU group. The results suggested that achieving low GHGE or EU diet might not lead to higher nutritional quality among Japanese. On the other hand, it may be possible that diet quality scores used in this study could not appropriately evaluate nutritional adequacy of the diet for the Japanese population as previously suggested.

\section{Conflict of Interest}

There is no conflict of interest 